\title{
Population-Based Tract-To-Region Connectome of the Human Brain and Its Hierarchical Topology
}

Fang-Cheng Yeh ( $\square$ frank.yeh@pitt.edu )

University of Pittsburgh

\section{Article}

Keywords: connectome, tractography, hierarchical clustering

Posted Date: November 30th, 2021

DOI: https://doi.org/10.21203/rs.3.rs-1083262/v1

License: (c) (i) This work is licensed under a Creative Commons Attribution 4.0 International License. Read Full License

Version of Record: A version of this preprint was published at Nature Communications on August 22nd, 2022. See the published version at https://doi.org/10.1038/s41467-022-32595-4. 


\section{Abstract}

Connectome maps region-to-region connectivities but does not inform which white matter pathways form the connections. Here we constructed the first population-based tract-to-region connectome to fill this information gap. The constructed connectome quantifies the population probability of a white matter tract innervating a cortical region. The results show that $\sim 85 \%$ of the tract-to-region connectome entries are consistent across individuals, whereas the remaining ( 15\%) have substantial individual differences requiring individualized mapping. Further hierarchical clustering on cortical regions revealed their parcellations into dorsal, ventral, and limbic networks based on the tract-to-region connective patterns. The clustering results on white matter bundles revealed the connectome-based categorization of fiber bundle systems in the association pathways. This new tract-to-region connectome provides insights into the connective topology between cortical regions and white matter bundles. The derived hierarchical relation further offers a connectome-based categorization of gray matter and white matter structures.

\section{Introduction}

Mapping the human connectome is the key to understanding how brain structure gives rise to functions and how brain diseases cause dysfunctions ${ }^{1,2}$. Studies have used structural or functional connectivity to quantify the region-to-region connectivity as the connectome ${ }^{3,4}$ and delineate the network topology of the nervous system. The network topology revealed by the brain connectome further informed the functional implications of cortical regions and enabled graphical theoretical analysis ${ }^{5}$. However, the conventional region-to-region connectome is agnostic of the role played by white matter pathways and does not indicate which pathways form the cortical connections. Consequently, for many neuroscience studies investigating region-to-region connectivity, the white matter is still a black box with much unknown that needs further exploration.

Here we mapped the first tract-to-region connectome to address this information gap. The connection probability between white matter pathways and cortical regions was evaluated on 1065 young adult subjects. For $m$ brain regions and $n$ white matter bundles, the tract-to-region connectome can be quantified by an $m$-by- $n$ matrix, where each matrix entry records the corresponding population probability of a white matter pathway innervating a cortical region. We leveraged several technical advances to construct the tract-to-region connectome (Fig. 1). The white matter bundles of the 1065 young adults were mapped using the recent advance in automated tractography ${ }^{6-10}$. The recognition of white matter bundles was accomplished by comparing the similarity of trajectories with an expert-vetted tractography ${ }^{11}$, and the irrelevant connections were removed to achieve high test-retest reliability ${ }^{12}$. Three tract-toregion connectome matrices were quantified using the Brodmann parcellation, Kleist parcellation ${ }^{13}$, and the Human Connectome Project's multimodal parcellation (HCP-MMP) ${ }^{14}$ atlases, respectively. The tractto-region information provided by this novel connectome could complete the circuit diagram for many structure-function models and inform the likelihood of a white matter lesion causing a functional deficit in the dysconnectome studies. Based on the tract-to-region connectome, we further applied hierarchical 
clustering to carry out connectome-based hierarchical clustering. The clustering results revealed the hierarchical relation of cortical regions and white matter pathways that informed their connectome-based categorization.

\section{Results}

\section{Population-based tractography of young adults}

We first examined the tractography of 1065 subjects in the ICBM152 space. Fig. 2a shows the voxel-wise probability of the association pathways, whereas Fig. $2 \mathrm{~b}$ shows the projection pathways. Each white matter tract is visualized by a population probability of $20 \%, 40 \%, 60 \%$, and $80 \%$, respectively. The probability was quantified by the percentage of subjects with the white matter bundle passing the ICBM152 space voxels. We detail our definition and abbreviations of white matter bundles in Suppl. Table 1. The tractography results shown in Fig. 2 are consistent with known neuroanatomy ${ }^{15}$ and existing tractography results $10,16,17$. The lateralization of left AF can be readily observed by its substantially larger volume.

Figure 3a visualizes all white matter bundles rendered by an iso-surface of $20 \%$ voxel-wise probability in the ICBM152 space. The AF and superior longitudinal fasciculus (SLF) in Fig. 3 show relatively broader coverage than those of the projection pathways such as corticospinal tract (CST), corticobulbar tract (CBT), optic radiation (OR), and fornix (F). This result can be explained by higher between-subject differences in $A F$ and SLF ${ }^{12}$. Fig. $3 b$ further shows coronal sections of tract probability. The maximum color saturation corresponds to $100 \%$ probability, whereas white color corresponds to $0 \%$ probability. The probabilities of association pathways are visualized to illustrate their anatomical relation and relative location. The results are consistent with a recent population-based tractography atlas ${ }^{17}$.

\section{Tract-to-region connectome}

The tract-to-region connectome based on Brodmann areas and Kleist parcellations are shown in Fig. 4a and Fig. 4b, respectively, whereas the one based on HCP-MMP parcellations is shown in Fig. 5 . The Brodmann, Kleist, and HCP-MMP parcellations have 39, 49, and 180 cortical regions. As shown in Fig. 4, the resulting connectivity matrices have 39-by-52 and 49-by-52 entries for Brodmann and Kleist atlases, whereas, in Fig. 5, there are 180-by-52 entries. Each row corresponds to a cortical region, and each column corresponds to a white matter tract. The population probability was quantified by checking the corresponding cortical region and white matter tract intersection in the ICBM152 space. The left half of the matrix is the connection probabilities in the left hemisphere, whereas the right half is the those in the right hemisphere. The probabilities are color-coded by red colors: the highest saturation corresponds to the highest probability $(100 \%)$, while the white color corresponds to the lowest probability $(0 \%)$. The entries with less than $5 \%$ connection probability are left blank to facilitate visualization. Most of the matrix entries in Brodmann (1733 out of 2028 entries, 85.45\%) and Kleist atlases (2164 out of 2548 entries, $84.93 \%$ ) have probability values greater than $95 \%$ or smaller than $5 \%$, meaning that these tract-to- 
region pairs are either connected or not connected in the majority of the study population. Around $15 \%$ of the matrix entries in Brodmann and Kleist atlases show probabilities between $5 \%$ and $95 \%$ due to substantial individual variation. Interestingly, although HCP-MMP parcellations have more parcellation regions (180 regions) than Brodmann and Kleist atlases (39 and 49 regions), it gives a remarkably similar figure. $86.50 \%$ of its matrix entries (8096 out of 9360 entries) also have probability values greater than $95 \%$ or smaller than $5 \%$. The tract-to-region connectome showed that the young adult population shares a similar connective pattern in $\sim 85 \%$ of the tract-to-region entries. The remaining $\sim 15 \%$ entries have substantial individual variations with population probability between $5 \%$ and $95 \%$, thus warranting individualized mapping.

We further examine arcuate fasciculus (AF) connections in the tract-to-region connectome using the Sankey flow diagrams shown in Fig. 6 . The diagrams use the HCP-MMP parcellation, and the color saturation scales with the population probability. The connective pattern shown in Fig. 6 is consistent with the conventional view that the left AF connects Wernicke's area in the superior temporal regions (red) and Broca's area in the inferior frontal cortex (orange). Furthermore, the diagrams also show more detailed connections of left AF to the caudal dorsolateral prefrontal cortex and the inferior parietal lobule ${ }^{18-22}$ as well as premotor/motor regions ${ }^{23}$. The lateralization of AF to frontoparietal (yellow), angular (green), and superior temporal regions (red) can also be seen by comparing Fig. 6a and 6b.

\section{Hierarchical relation of cortical regions}

Figure 7 shows the similarity matrices and the derived hierarchical relations of the cortical regions defined by Brodmann (Fig. 7a) and Kleist atlases (Fig. 7b), whereas the results for HCP-MMP is shown in Fig. 8. The column and row orders of the matrices are reordered based on clustering results to facilitate inspection. The dendrograms on the top of Fig. 7 and 8 show the hierarchical relation of the cortical regions and the vertical distance scales by the cost for merging. Overall, Fig. 7 and 8 show a consistent result, with cortical regions categorized into dorsal, ventral, and limbic networks. Although differences can be observed at each atlas, the dorsal network includes most frontal (excluding prefrontal) and parietal regions, whereas the ventral network includes temporal and occipital regions. The limbic network is constituted of prefrontal, insula, and upper cingulum regions. In Brodmann atlas (Fig. 7a), its dorsal network further includes the superior temporal gyrus. In contrast, the dorsal network in Kleist atlas (Fig. 7b) and HCP-MMP atlases (Fig. 8) only include a small posterior section of the superior temporal gyrus. The discrepancy is likely due to more detailed parcellation in Kleist and HCP-MMP at areas 22, 39, and 40 . This suggests the necessity of using a detailed parcellation to avoid under-clustering. The detailed parcellation in the HCP-MMP atlas allows for revealing the subnetworks under the dorsal network, including frontal (orange colored), inferior parietal (yellowish and light green), and superior parietal (cyan) subnetworks (Suppl. Fig. 1). Similarly, the HCP-MMP shows structures under the ventral networks, including occipital (purple and light blue), inferior temporal (magenta), superior temporal (light red) subnetworks (Suppl. Fig. 2). The limbic networks are primarily consistent across Brodmann, Kleist, and HCP-MMP atlases. The including regions cover prefrontal regions and cingulum that bridges between dorsal and ventral networks. More detailed subnetworks based on HCP-MMP are shown in Suppl. Fig. 3. 


\section{Hierarchical relation of white matter bundles}

Figure 9 further shows the similarity matrix between the association pathways (Fig. 9a), and the dendrogram illustrates the hierarchical clustering results (Fig. 9b) based on the HCP-MMP tract-to-region connectome. The left and right hemispheres show highly similar hierarchical relations that groups association pathways into four systems, including the arcuate system (purple), anterior ventral system (red), posterior ventral system (cyan), and cingulum system (green). The first system includes AF, SLF II, SLF III, and FAT. These pathways all connect to Broca's area are known to be associated with language functions. Fig. $3 \mathrm{~b}$ also shows supporting results that SLF II and III are closely neighboring the AF ( $Y=-11$, $Y=-19$, and $Y=-27)$. The second system includes MdLF, TPAT, VOF, and ILF. TPAP is also commonly known as the posterior AF. The close relation between VOF and ILF suggests that VOF can be viewed as a component of ILF. The third system includes UF and IFOF, and both are characterized by their frontal connection from the temporal and occipital lobes, respectively. The fourth system includes all cingulum pathways and SLF I, likely due to the fact that SLF I is closely adjacent to cingulum at $(Y=-3$ and $Y=-11)$ and entirely separated from SLF II and III by FAT (Fig. 3b). This result suggests that the SLF I could be considered as part of the cingulum system.

\section{Discussion}

Here we quantify the tract-to-region connectome in the young adult population. The constructed matrices provide a resource for both neuroscience and clinical studies to evaluate the probability of a white matter tract connecting to a cortical region. From this tract-to-region connectome, we further derived hierarchical relations between cortical regions to understand the topological relations. The overall result of tract-toregion hierarchical relation revealed dorsal, ventral, and limbic systems, especially using more detailed parcellation atlases such as the HCP-MMP. The revealed dorsal, ventral, and limbic networks can be applied to many existing functional models. The dorsal system includes most frontal lobe (excluding prefrontal) and parietal lobe, whereas the ventral system includes the temporal lobe and occipital lobe. The dorsal and ventral subnetworks shared many similarities with the existing dual-stream model in language and visual functions ${ }^{24-27}$. The topological relation shown in this study disclosed the role of white matter pathways to supplement existing fMRI-based localization of cortical regions.

We also derived the hierarchical relation between white matter bundles to show the categorical relation of the association pathways. While many studies have been conducted to cluster white matter tracts ${ }^{7,28-32}$, the clustering methods were based on tract trajectories and did not consider the connective pattern with the cortical regions. The clustering in this study did not use tract trajectories. The results were derived from their connective pattern with cortical regions to offer a different view toward the recent dispute of neuroanatomy nomenclature, particularly in the naming and segmentation of arcuate fasciculus and superior longitudinal fasciculus ${ }^{33}$. Our hierarchical clustering results suggest that SLF II and SLF III are closely related to AF, whereas SLF I is closely related to the cingulum. These clustering results support the naming convention by Catani et al. ${ }^{19}$ that categorized SLF II and III as the subcomponent of the AF. The 
relation between the SLF I and the cingulum has also been proposed previously: Wang et al. ${ }^{34}$ suggested that the SLF I should be viewed as part of the cingulum system. Based on our tract-to-region connectome, it is likely that damaging SLF I may not lead to the same function deficit as SLF II and III. Therefore, the SLF I could be reasonably renamed as the frontal-parietal component of the cingulum. It is noteworthy that the current SLF I, II, III definitions could be sourced back to non-human primates studies ${ }^{35}$. Our tractto-region connectome shows a different perspective: the SLF II and III could be viewed as subcomponents of AF, whereas SLF I could be viewed as a subcomponent of the cingulum. Further functional or lesionbased studies are needed to support or refute this categorization conjecture.

In comparison with the tract-to-region connectome, the existing region-to-region connectome predominately focused on region-to-region connections. The graph models based on connectome often simply the role of the white matter bundles as a single "edge" in the network model, although neuroanatomical evidence has shown that brain regions in both human and non-human primates can be connected through more than one route ${ }^{36}$. As a result, the clustering results are substantially different between the tract-to-region and region-to-region connectome: the clustering based on region-to-region connectome concerns whether the cortical regions are closely connected ${ }^{37,38}{ }^{\text {, whereas the clustering }}$ based on tract-to-region connectome concerns whether the cortical regions share similar white matter connections. One of the notable differences is that region-to-region connectome tends to group frontal and prefrontal regions due to their strong connections through short association pathways ${ }^{38,39}$, but the results from tract-to-region connectome shown in this stud separated prefrontal and frontal regions due to their distinctly different connections with the limbic system. The difference in clustering context will lead to entirely different results and application scenarios that answer different neuroscience questions.

In addition to differences in clustering results, the region-to-region connectome falls short of illustrating the association between cortical regions and white matter pathways. This limitation becomes obvious in lesion-symptom mapping studies of aphasia: damaging Broca's area does not necessarily lead to Broca's aphasia 40,41 , and in contrast, lesions involving the anterior segment of the left arcuate fasciculus is a strong symptom predictor ${ }^{42}$. Thus, the functional role of white matter connections cannot be ignored, and robust prediction of brain dysfunction requires tract-to-region information. Studies have utilized fiber tracking to probe into the effect of white matter lesions to bridge the gap between the connective information between white matter bundles and cortical regions ${ }^{43-45}$. For those studies, the tract-to-region connectome can provide a population-based reference to understand the relation between cortical regions and white matter bundles. The rich information between the white matter tract and cortical regions are critical for clinical study of "dysconnectome," as recent studies suggested that cortical regions themselves are not sufficient enough to explain functional deficits ${ }^{46}$ and the role of white matter pathways should be considered ${ }^{40,43}$. Our tract-to-region connectome can be a stepping stone to explore the lesion-symptom relation and understand how the connective pattern alters after a brain injury.

\section{Materials And Methods}


The diffusion MRI data of 1065 subjects (Fig. 1a) were acquired from the Human Connectome Project database (WashU consortium) ${ }^{2}$. The age range was 22- to 37-year-old, and the average age was 28.75 . The data were acquired using a multishell diffusion scheme with three b-values at 1000, 2000, and 3000 $\mathrm{s} / \mathrm{mm}^{2}$ with 90 sampling directions for each shell. The spatial resolution was $1.25 \mathrm{~mm}$ isotropic. The detailed acquisition parameters are listed in the consortium paper ${ }^{2}$. The preprocessed data were used and further corrected for gradient nonlinearity.

\section{Diffusion MRI reconstruction}

The diffusion data were linearly rotated to align with the ac-pc line of the ICBM152 space and simultaneously interpolated at $1 \mathrm{~mm}$ using a cubic spline. The b-table was also rotated accordingly. The rotated data were then reconstructed using generalized q-sampling imaging ${ }^{47}$ with a diffusion sampling length ratio of 1.7. An automatic quality control routine was adopted to check the b-table orientation and ensure its accuracy ${ }^{48}$. The reconstruction results (Fig. 1b) then guided further automated tractography.

\section{Automated tractography}

For each subject, 52 white matter bundles were mapped using automated tractography that combined deterministic fiber tracking algorithm ${ }^{49}$ with parameter saturation and randomized parameters ${ }^{12}$ and 20 iterations of topology-informed pruning ${ }^{50}$. Trajectory recognition was based on an updated populationaveraged tractography atlas ${ }^{11}$, and the maximum allowed Hausdorff distance (tolerance distance) was assigned by 16 and increased to 18 and $20 \mathrm{~mm}$ if it yielded no result. The white matter bundles of each subject were then output to the ICBM152 2009 nonlinear space (Fig. 1C). The analysis was conducted on the Pittsburgh Supercomputing Center provided through the Extreme Science and Engineering Discovery Environment (XSEDE) resource ${ }^{51}$. The tractography result for each subject and each white matter tract are shared on http://brain.labsolver.org.

\section{Brain parcellations and tract-to-region connectome}

We examined whether a white matter bundle is connected to a region in each of the 1065 subjects. The trajectories of white matter bundles in the ICBM152 space were examined with the Brodmann, Kleist, and HCP-MMP atlases to derive the population-based tract-to-region connectome (Fig. 1d). We used the ICBM152 space version of newly reconstructed Brodmann and Kleist atlases ${ }^{13}$. On the other hand, the ICBM152 space version of the HCP-MMP atlas was obtained from

https://neurovault.org/collections/1549/ (asymmetric, improved reconstruction) and further inspected for each cortical region to manually remove the cross-sulci leakage using DSI Studio. The revised version of the HCP-MMP atlas was shared with the DSI Studio package and is publicly available at http://dsistudio.labsolver.org. For each subject, a binary tract-to-region connection matrix was obtained with each of the three parcellation atlases by calculating the intersection between the voxel-wise mapping of white matter bundles and cortical regions (Fig. 1e). The matrices of 1065 subjects were then aggregated to 
compute the population probability of the tract-to-region connection. Three matrices were generated for Brodmann, Kleist, and HCP-MMP atlases, respectively. The tract-to-region connectome can be downloaded from http://brain.labsolver.org

\section{Hierarchical clustering}

We used row vectors of the tract-to-region matrices as the feature vectors to derive the hierarchical relation between cortical regions (Fig. 1f). The similarity matrices between each region pair were calculated using nonparametric Spearman's rank correlation. The hierarchical clustering was conducted using weighted average distance ${ }^{52}$ provided by the linkage function in MATLAB to avoid the high variability drawback of simple single linkage clustering. For each cortical parcellation atlas (Brodmann, Kleist, HCP-MMP), a dendrogram was generated to reveal the hierarchical relation of the cortical regions. The hierarchical clustering for white matter bundles was conducted using column vector of the HCP-MMP connectome matrices (Fig. 1g). The clustering routine also used weighted average distance to generate the dendrogram to reveal the hierarchical relation of white matter bundles.

\section{Data availability}

The analysis tool DSI Studio and its source code are available at http://dsi-studio.labsolver.org. The population-based tractography and tract-to-region connectome are publicly available at http://brain.labsolver.org.

\section{Declarations}

\section{Acknowledgments}

This work used the Extreme Science and Engineering Discovery Environment (XSEDE), which is supported by National Science Foundation grant number ACl-1548562. The computation resources were allocated under TG-CIS200026.

\section{References}

1 Akil, H., Martone, M. E. \& Van Essen, D. C. Challenges and opportunities in mining neuroscience data. Science 331, 708-712, doi:331/6018/708 [pii] 10.1126/science.1199305 (2011).

2 Glasser, M. F. et al. The Human Connectome Project's neuroimaging approach. Nat Neurosci 19, 1175-1187, doi:10.1038/nn.4361 (2016).

3 Sporns, O., Tononi, G. \& Kotter, R. The human connectome: A structural description of the human brain. PLoS Comput Bio/ 1, e42, doi:10.1371/journal.pcbi.0010042 (2005).

4 Biswal, B. B. et al. Toward discovery science of human brain function. Proc Natl Acad Sci U S A 107, 4734-4739, doi:10.1073/pnas.0911855107 (2010). 
5 Bullmore, E. \& Sporns, O. Complex brain networks: graph theoretical analysis of structural and functional systems. Nat Rev Neurosci 10, 186-198, doi:10.1038/nrn2575 (2009).

6 O'Donnell, L. J. et al. Automated white matter fiber tract identification in patients with brain tumors. Neurolmage: Clinical 13, 138-153 (2017).

7 Garyfallidis, E. et al. Recognition of white matter bundles using local and global streamline-based registration and clustering. Neuroimage 170, 283-295, doi:10.1016/j.neuroimage.2017.07.015 (2018).

8 Rheault, F. et al. Bundle-specific tractography with incorporated anatomical and orientational priors. Neurolmage 186, 382-398 (2019).

9 Zollei, L., Jaimes, C., Saliba, E., Grant, P. E. \& Yendiki, A. TRActs constrained by UnderLying INfant anatomy (TRACULInA): An automated probabilistic tractography tool with anatomical priors for use in the newborn brain. Neuroimage 199, 1-17, doi:10.1016/j.neuroimage.2019.05.051 (2019).

10 Zhang, F. et al. An anatomically curated fiber clustering white matter atlas for consistent white matter tract parcellation across the lifespan. Neuroimage 179, 429-447, doi:10.1016/j.neuroimage.2018.06.027 (2018).

11 Yeh, F. C. et al. Population-averaged atlas of the macroscale human structural connectome and its network topology. Neuroimage 178, 57-68, doi:10.1016/j.neuroimage.2018.05.027 (2018).

12 Yeh, F. C. Shape analysis of the human association pathways. Neuroimage 223, 117329, doi:10.1016/j.neuroimage.2020.117329 (2020).

13 Pijnenburg, R. et al. Myelo- and cytoarchitectonic microstructural and functional human cortical atlases reconstructed in common MRI space. Neuroimage 239, 118274, doi:10.1016/j.neuroimage.2021.118274 (2021).

14 Glasser, M. F. et al. A multi-modal parcellation of human cerebral cortex. Nature 536, 171-178, doi:10.1038/nature18933 (2016).

15 Catani, M. \& de Schotten, M. T. Atlas of human brain connections. (Oxford University Press, 2012).

16 Thiebaut de Schotten, M. et al. Atlasing location, asymmetry and inter-subject variability of white matter tracts in the human brain with MR diffusion tractography. Neuroimage 54, 49-59, doi:10.1016/j.neuroimage.2010.07.055 (2011).

17 Hansen, C. B. et al. Pandora: 4-D White Matter Bundle Population-Based Atlases Derived from Diffusion MRI Fiber Tractography. Neuroinformatics, doi:10.1007/s12021-020-09497-1 (2020).

18 Bernal, B. \& Ardila, A. The role of the arcuate fasciculus in conduction aphasia. Brain 132, 23092316 (2009). 
19 Catani, M., Jones, D. K. \& ffytche, D. H. Perisylvian language networks of the human brain. Ann Neuro/ 57, 8-16, doi:10.1002/ana.20319 (2005).

20 Glasser, M. F. \& Rilling, J. K. DTI tractography of the human brain's language pathways. Cerebral cortex 18, 2471-2482 (2008).

21 Rilling, J. K. et al. The evolution of the arcuate fasciculus revealed with comparative DTI. Nat Neurosci 11, 426-428, doi:10.1038/nn2072 (2008).

22 Saur, D. et al. Ventral and dorsal pathways for language. Proc Natl Acad Sci U S A 105, 1803518040, doi:10.1073/pnas.0805234105 (2008).

23 Bernal, B. \& Ardila, A. The role of the arcuate fasciculus in conduction aphasia. Brain 132, 23092316, doi:10.1093/brain/awp206 (2009).

24 Fridriksson, J. et al. Revealing the dual streams of speech processing. Proceedings of the National Academy of Sciences 113, 15108-15113 (2016).

25 Milner, A. D. \& Goodale, M. A. Two visual systems re-viewed. Neuropsychologia 46, 774-785 (2008).

26 Rauschecker, J. P. \& Tian, B. Mechanisms and streams for processing of "what" and "where" in auditory cortex. Proceedings of the National Academy of Sciences 97, 11800-11806 (2000).

27 Mishkin, M., Ungerleider, L. G. \& Macko, K. A. Object vision and spatial vision: two cortical pathways. Trends in neurosciences 6, 414-417 (1983).

28 Siless, V., Chang, K., Fischl, B. \& Yendiki, A. AnatomiCuts: Hierarchical clustering of tractography streamlines based on anatomical similarity. Neuroimage 166, 32-45, doi:10.1016/j.neuroimage.2017.10.058 (2018).

29 Guevara, P. et al. Robust clustering of massive tractography datasets. Neuroimage 54, 1975-1993, doi:S1053-8119(10)01320-0 [pii]

10.1016/j.neuroimage.2010.10.028 (2011).

30 Jin, Y. et al. Automatic clustering of white matter fibers in brain diffusion MRI with an application to genetics. Neuroimage 100, 75-90, doi:10.1016/j.neuroimage.2014.04.048 (2014).

31 Maddah, M., Mewes, A. U., Haker, S., Grimson, W. E. \& Warfield, S. K. Automated atlas-based clustering of white matter fiber tracts from DTMRI. Med Image Comput Comput Assist Interv 8, 188-195 (2005).

32 Wang, Q., Yap, P. T., Wu, G. \& Shen, D. Application of neuroanatomical features to tractography clustering. Hum Brain Mapp, doi:10.1002/hbm.22051 (2012). 
33 Dick, A. S. \& Tremblay, P. Beyond the arcuate fasciculus: consensus and controversy in the connectional anatomy of language. Brain 135, 3529-3550, doi:10.1093/brain/aws222 (2012).

34 Wang, X. et al. Subcomponents and connectivity of the superior longitudinal fasciculus in the human brain. Brain Struct Funct 221, 2075-2092, doi:10.1007/s00429-015-1028-5 (2016).

35 Petrides, M. \& Pandya, D. N. Projections to the frontal cortex from the posterior parietal region in the rhesus monkey. J Comp Neuro/ 228, 105-116, doi:10.1002/cne.902280110 (1984).

36 Ghulam-Jelani, Z. et al. Redundancy circuits of the commissural pathways in human and rhesus macaque brains. Hum Brain Mapp 42, 2250-2261, doi:10.1002/hbm.25363 (2021).

37 Sohn, Y., Choi, M. K., Ahn, Y. Y., Lee, J. \& Jeong, J. Topological cluster analysis reveals the systemic organization of the Caenorhabditis elegans connectome. PLoS Comput Biol 7, e1001139, doi:10.1371/journal.pcbi.1001139 (2011).

38 Akiki, T. J. \& Abdallah, C. G. Determining the Hierarchical Architecture of the Human Brain Using Subject-Level Clustering of Functional Networks. Sci Rep 9, 19290, doi:10.1038/s41598-019-55738-y (2019).

39 Raut, R. V., Snyder, A. Z. \& Raichle, M. E. Hierarchical dynamics as a macroscopic organizing principle of the human brain. Proc Natl Acad Sci U S A 117, 20890-20897, doi:10.1073/pnas.2003383117 (2020).

40 Gajardo-Vidal, A. et al. Damage to Broca's area does not contribute to long-term speech production outcome after stroke. Brain 144, 817-832 (2021).

41 Ardila, A., Bernal, B. \& Rosselli, M. Why Broca's area damage does not result in classical Broca's aphasia. Frontiers in human neuroscience 10, 249 (2016).

42 Fridriksson, J., Guo, D., Fillmore, P., Holland, A. \& Rorden, C. Damage to the anterior arcuate fasciculus predicts non-fluent speech production in aphasia. Brain 136, 3451-3460, doi:10.1093/brain/awt267 (2013).

43 Thiebaut de Schotten, M., Foulon, C. \& Nachev, P. Brain disconnections link structural connectivity with function and behaviour. Nat Commun 11, 5094, doi:10.1038/s41467-020-18920-9 (2020).

44 Griffis, J. C., Metcalf, N. V., Corbetta, M. \& Shulman, G. L. Lesion Quantification Toolkit: A MATLAB software tool for estimating grey matter damage and white matter disconnections in patients with focal brain lesions. Neuroimage Clin 30, 102639, doi:10.1016/j.nicl.2021.102639 (2021).

45 Greene, C. et al. Finding maximally disconnected subnetworks with shortest path tractography. Neuroimage Clin 23, 101903, doi:10.1016/j.nicl.2019.101903 (2019). 
46 Salvalaggio, A., De Filippo De Grazia, M., Zorzi, M., Thiebaut de Schotten, M. \& Corbetta, M. Poststroke deficit prediction from lesion and indirect structural and functional disconnection. Brain 143, 21732188, doi:10.1093/brain/awaa156 (2020).

47 Yeh, F. C., Wedeen, V. J. \& Tseng, W. Y. Generalized q-sampling imaging. IEEE Trans Med Imaging 29, 1626-1635, doi:10.1109/TMI.2010.2045126 (2010).

48 Schilling, K. G. et al. A fiber coherence index for quality control of B-table orientation in diffusion MRI scans. Magn Reson Imaging 58, 82-89, doi:10.1016/j.mri.2019.01.018 (2019).

49 Yeh, F. C., Verstynen, T. D., Wang, Y., Fernandez-Miranda, J. C. \& Tseng, W. Y. Deterministic diffusion fiber tracking improved by quantitative anisotropy. PLoS ONE 8, e80713, doi:10.1371/journal.pone.0080713

PONE-D-13-26801 [pii] (2013).

50 Yeh, F. C. et al. Automatic Removal of False Connections in Diffusion MRI Tractography Using Topology-Informed Pruning (TIP). Neurotherapeutics 16, 52-58, doi:10.1007/s13311-018-0663-y (2019).

51 Towns, J. et al. XSEDE: accelerating scientific discovery. Computing in science \& engineering 16, 62-74 (2014).

52 Sokal, R. R. A statistical method for evaluating systematic relationships. Univ. Kansas, Sci. Bull. 38, 1409-1438 (1958).

\section{Figures}

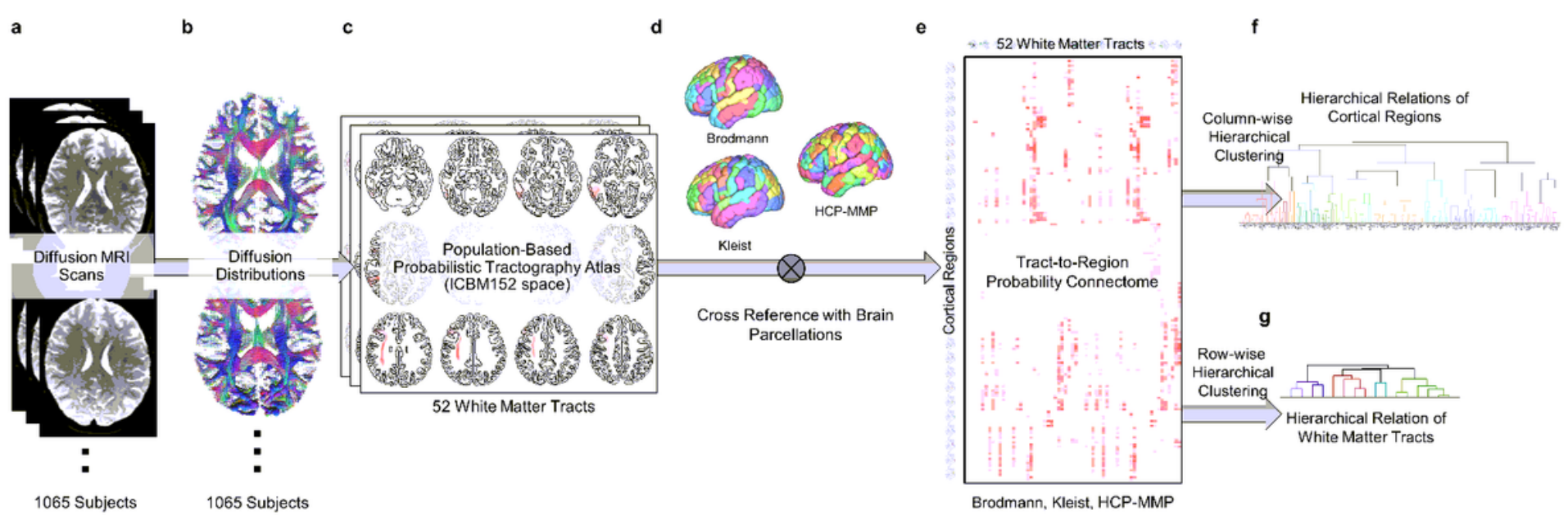

\section{Figure 1}

The processing flow to construct a population-based tractography connectome and derive its hierarchical relation. (a) The diffusion MRI data of 1065 subjects were used. (b) The data were reconstructed to 
calculate the diffusion distribution for fiber tracking. (c) For each subject, 52 white matter bundles were mapped using automated tractography. The track recognition was based on trajectory similarity with a tractography atlas without using the cortical parcellations. (d) The tracking results were aggregated to construct a population-based probability atlas of 52 white matter pathways. (e) 180 cortical regions from multimodal cortical parcellations and the white matter trajectories of each subject were cross-referenced.

(f) The results from each subject were accumulated to construct a 52-by-180 tract-region connectome based on population probability. (g) Hierarchical clustering was applied to the row vectors of the connectome to derive the hierarchical relation of white matter bundles. (h) Hierarchical clustering was applied to the column vectors to derive the hierarchical relation of cortical regions. 
a

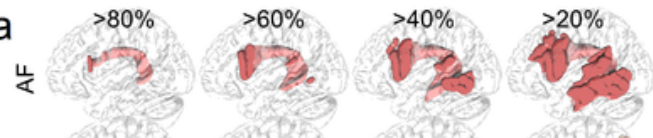

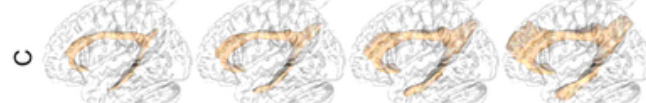

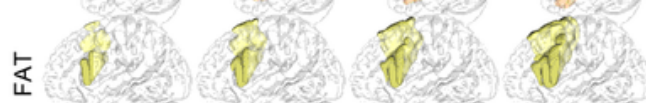

".

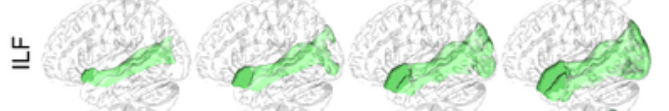

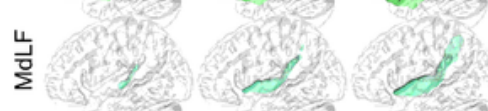
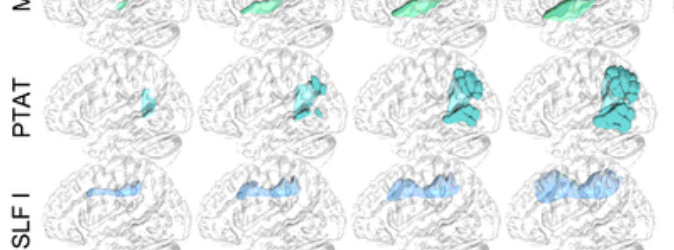

$=$ in

$\equiv$ mes ne nd n?

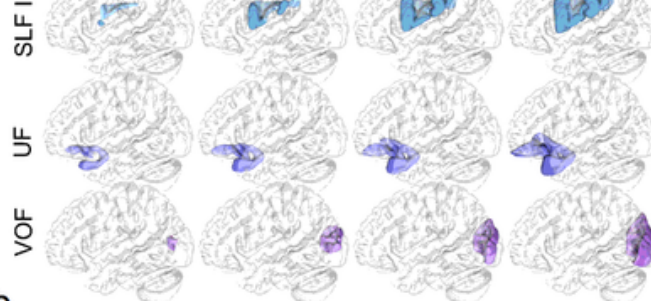

b

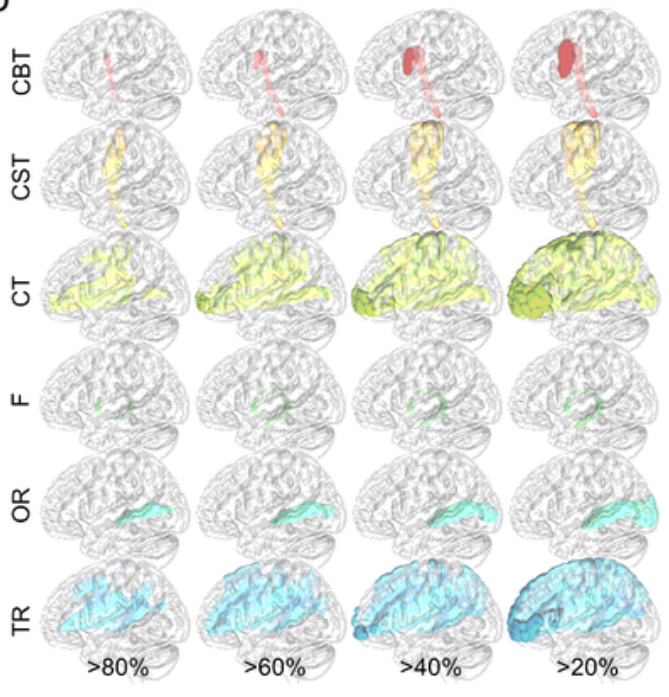

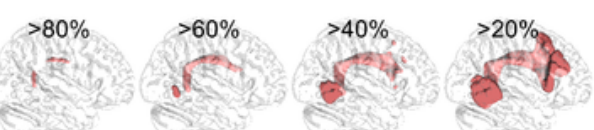

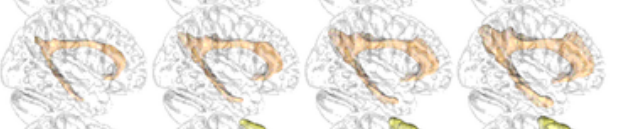
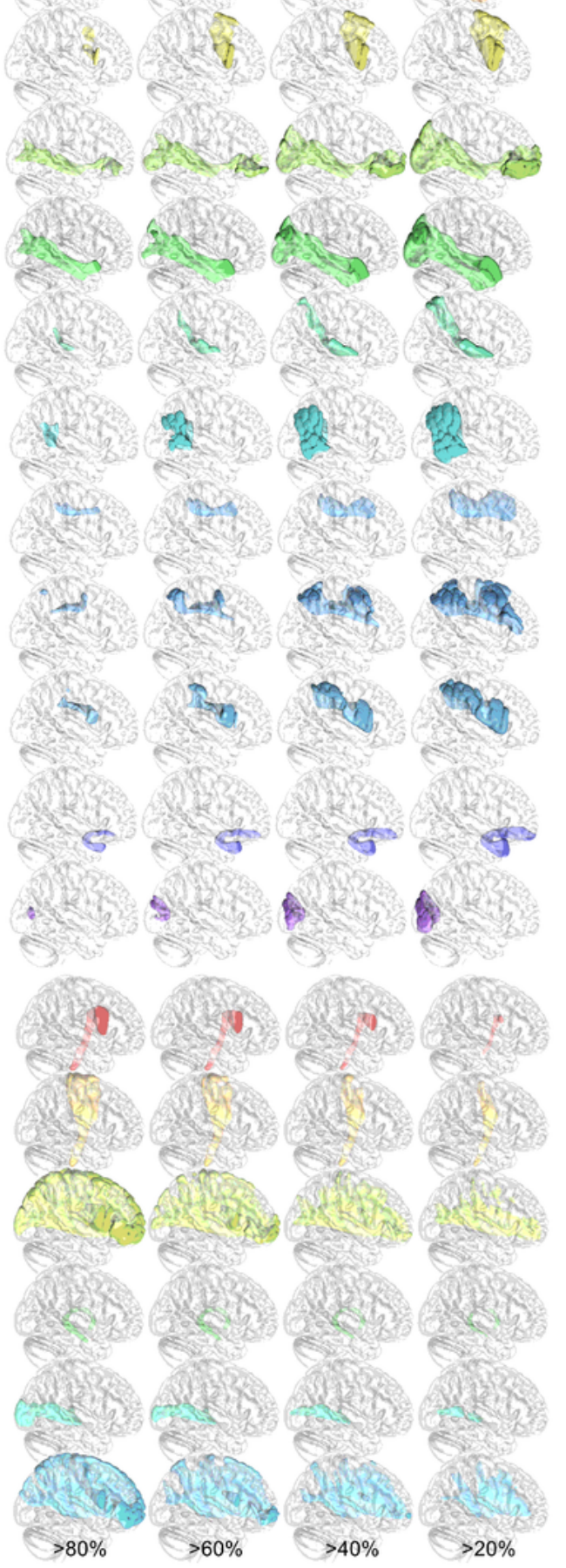

\section{Figure 2}

Probabilistic tractography atlas of white matter pathways visualized at population probability of $20 \%$, $40 \%, 60 \%$, and $80 \%$. (a) Association pathways are visualized at different population probabilities. (b) Projection pathways are visualized at different population probabilities. 
a
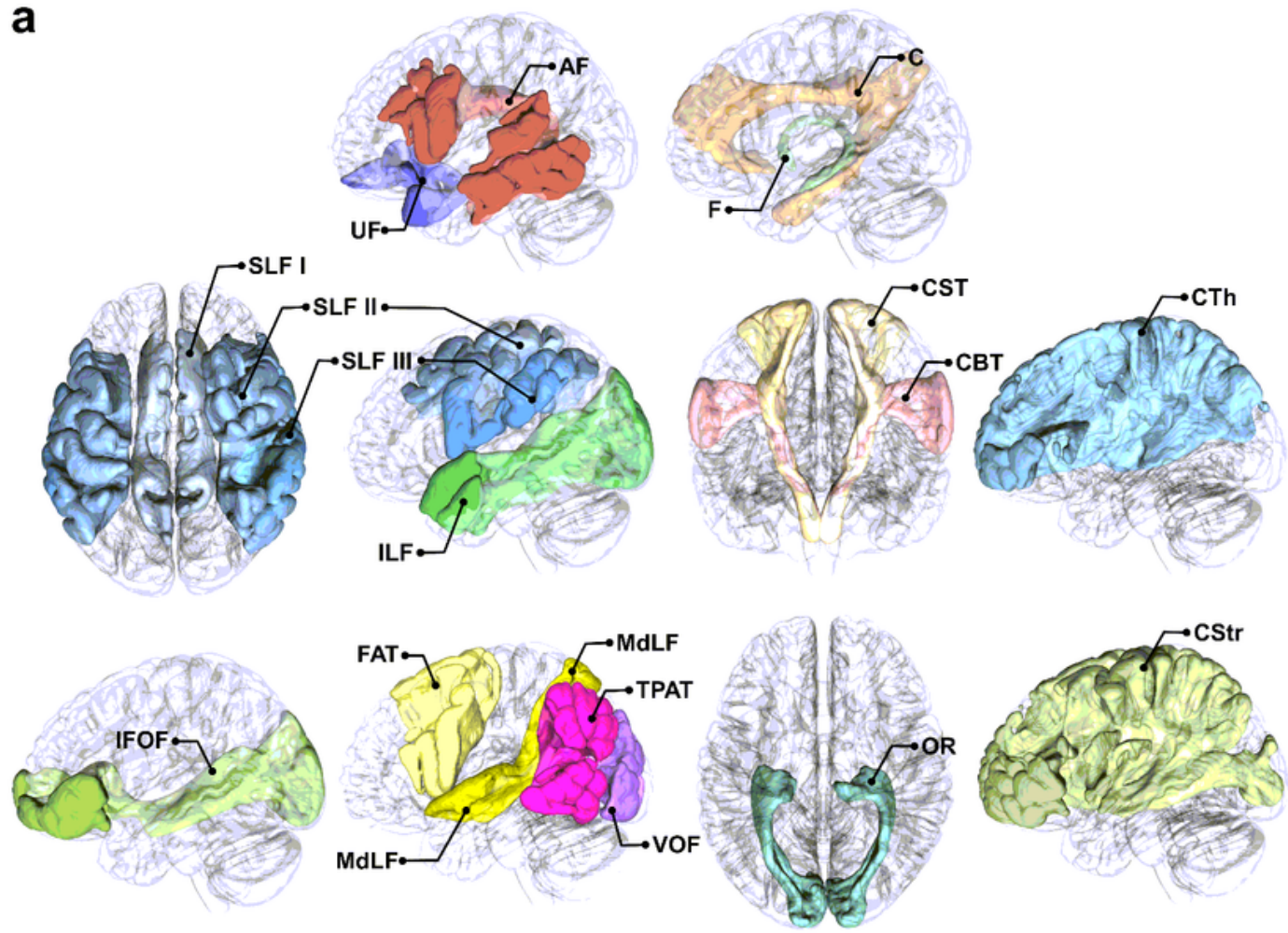

b
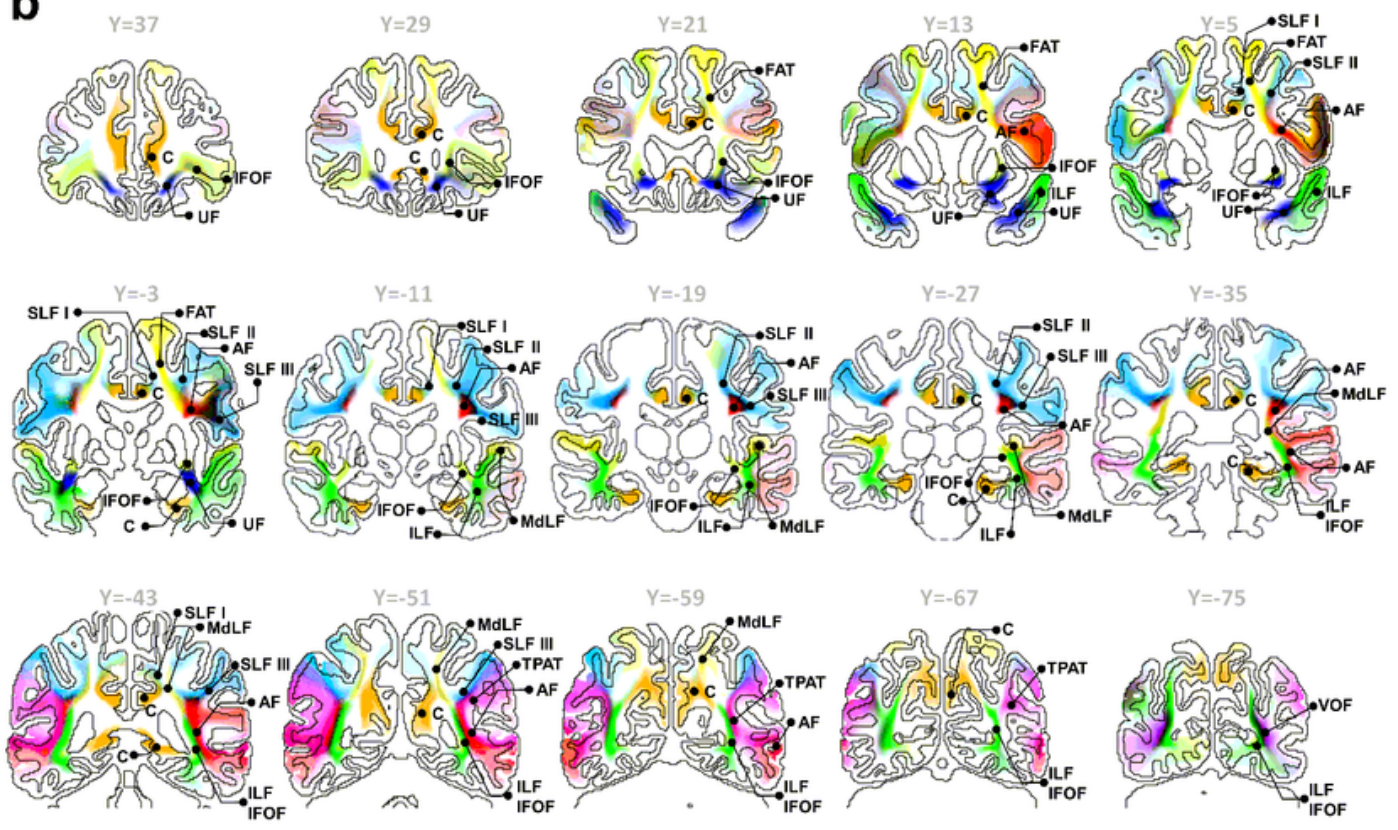

\section{Figure 3}

Overview of the population-based tractography atlas in 3D rendering and slice-wise coronal sections. (a) White matter pathways are visualized using $20 \%$ population probability. (b) Coronal sections of association pathways in the ICBM152 space. The color intensity scales with the population probability of the white matter bundles in the young adult population. 

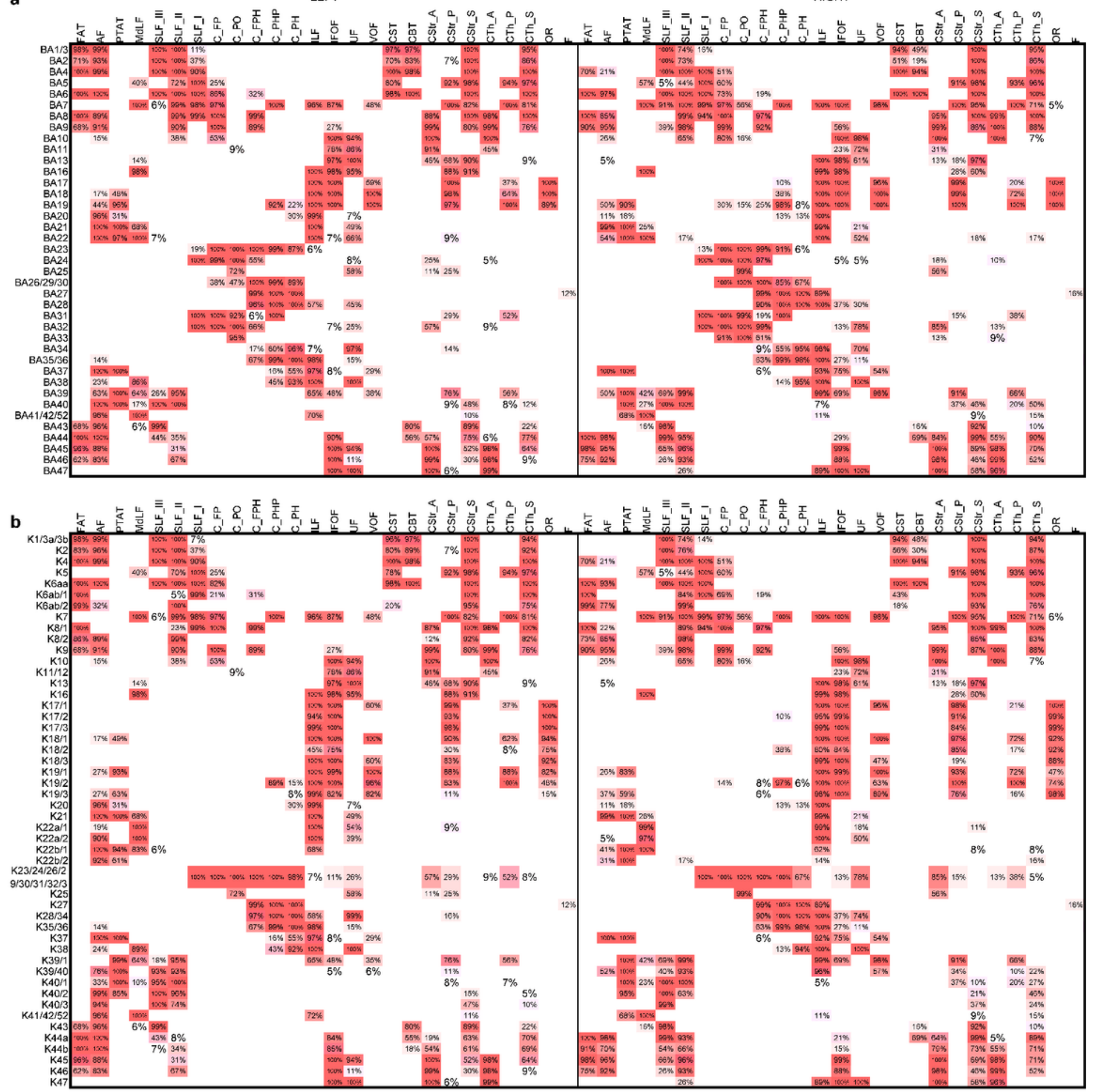

Figure 4

The probabilistic tract-to-region connectome matrices derived from (a) Brodmann and (b) Kleist brain parcellations. The rows of the matrices correspond to each brain regions defined by cortical parcellations, whereas the columns correspond to each white matter bundles. Each tract-region pair shows the population probability quantified from 1065 young adults. Probability values lower than $5 \%$ were left blank to facilitate inspection. 


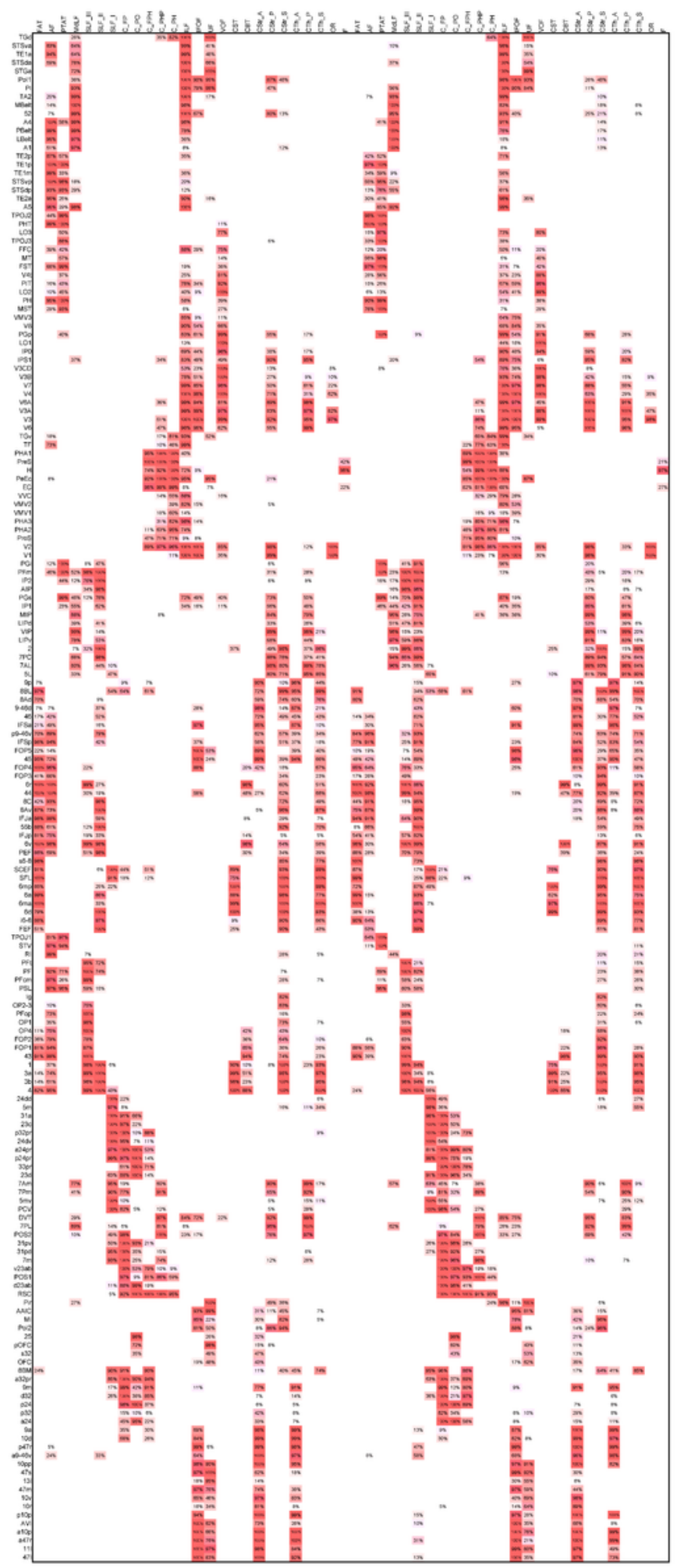

Figure 5

The probabilistic tract-to-region connectome matrices derived from the HCP multimodal parcellation. The 180 rows of the matrices correspond to each brain region defined by the HCP cortical parcellations, whereas the columns correspond to each white matter bundle. Each tract-region pair shows the population probability quantified from 1065 young adults. Probability values lower than $5 \%$ were left blank to facilitate inspection. 


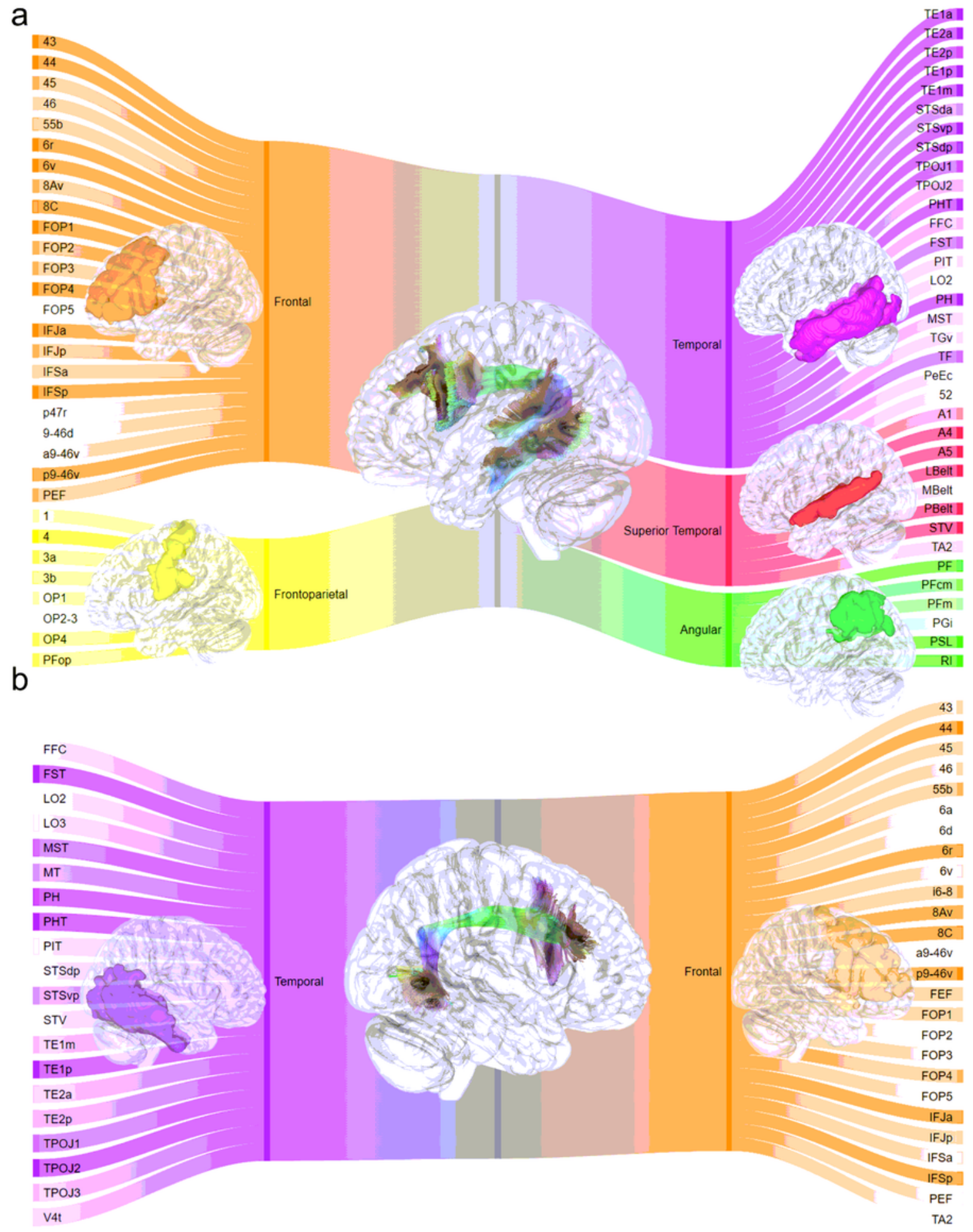

\section{Figure 6}

The tract-to-region connective pattern of the (a) left and (b) right arcuate fasciculus shown by Sankey flow diagrams. The diagrams are based the population probability calculated from the tract-to-region connectome in Fig. 5. The color saturation scales with the connection probability. The left arcuate fasciculus shows substantially lateralized connections to frontalparietal (yellow), angular (green), and superior temporal regions (red). 

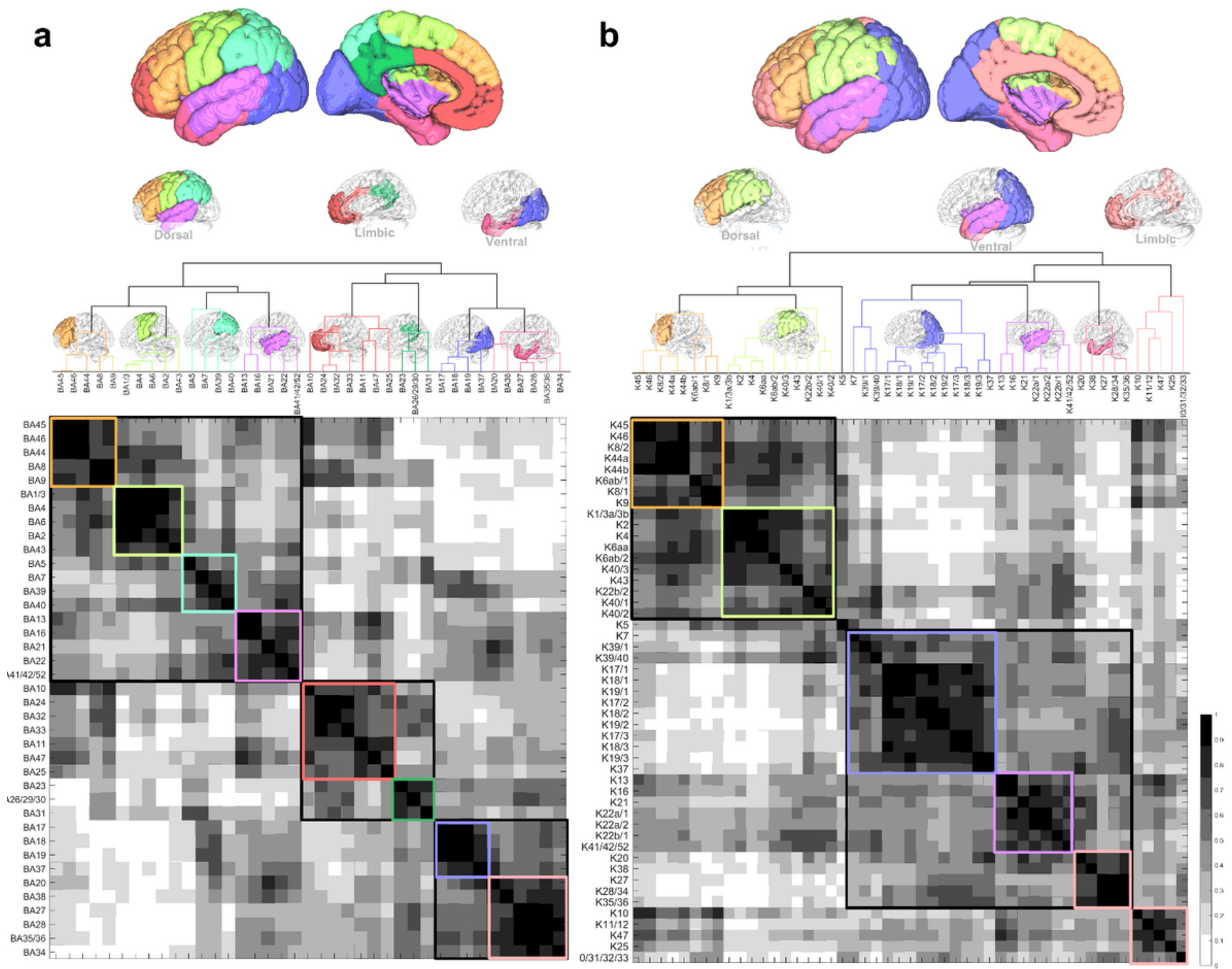

\section{Figure 7}

Similarity matrices between cortical regions and derived dendrograms based on (a) Brodmann and (b) Kleist parcellations. The similarity matrices were calculated by nonparametric Spearman correlation between the row vectors of the connectome matrices. The hierarchical relation of cortical areas is then visualized using dendrograms computed by using hierarchical clustering. The vertical distances in the dendrograms are scaled with the clustering cost. Both dendrograms show grossly consistent results revealing three major clusters: the limbic, dorsal, and ventral networks. 


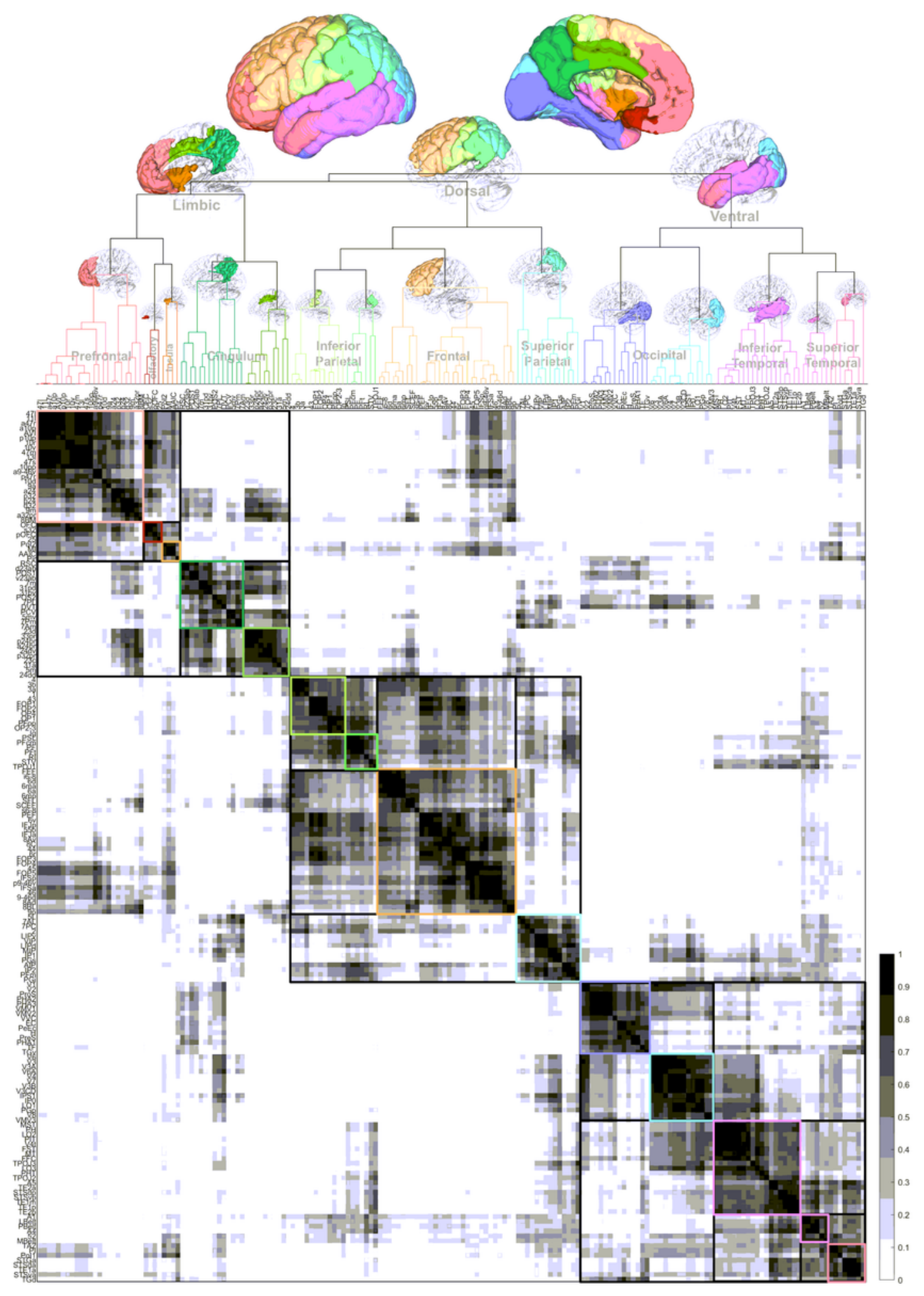

\section{Figure 8}

Similarity matrix between cortical regions and its derived dendrogram based on HCP multimodal parcellation. Consistent with the previous figure's results derived from Brodmann and Kleist parcellations, the cortical regions can be clustered into limbic, dorsal, and ventral networks. The limbic network includes the limbic system, prefrontal cortex, olfactory cortex, and insula. The dorsal network includes most of the remaining frontal lobe, parietal lobe, and part of the superior temporal gyrus, whereas the ventral network 
includes most of the temporal and occipital lobe. Each network has its downstream hierarchical structures of the subcomponent networks.
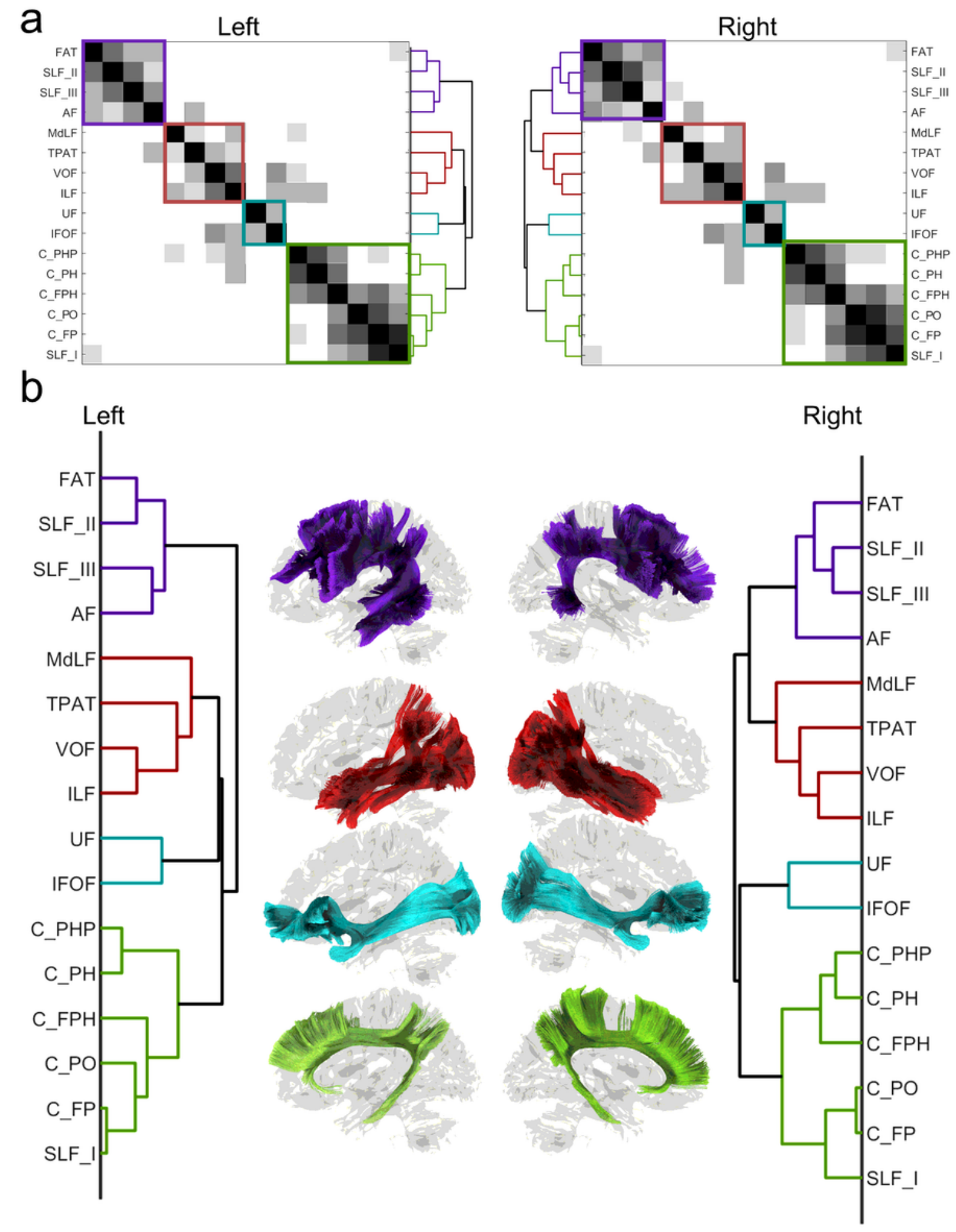

Figure 9

Similarity matrices between association pathways and their derived dendrograms based on HCP multimodal parcellation. The similarity matrices were calculated by nonparametric Spearman correlation between the column vectors of the connectome matrix. The hierarchical relation of cortical areas is then 
visualized using dendrograms computed by using hierarchical clustering. The horizontal distance in the dendrograms scales with the clustering cost. Both dendrograms show four categories of association pathways on both hemispheres, including the cingulum system (green), posterior ventral system (cyan), anterior ventral system (red), arcuate system (purple).

\section{Supplementary Files}

This is a list of supplementary files associated with this preprint. Click to download.

- SupTable1.xlsx

- sfig1.tif

- sfig2.tif

- sfig3.tif 